\title{
Recurrent thrombotic occlusions of arteries and veins caused by intravascular metastatic adenocarcinoma
}

\author{
M Levi, C Bronkhorst, L A Noorduyn, J Vreeken
}

\begin{abstract}
The occurrence of unexplained, rapidly recurring occlusions of arteries and veins in a previously healthy young woman is described. Post mortem examination showed no macroscopic abnormalities but revealed microscopic metastatic adenocarcinoma with remarkable intravascular localisation of the malignant cells. Whereas highly sensitive markers for the existence of systemic activation of blood coagulation remained within the normal range, it is suggested that specific characteristics of the tumour cells may have been responsible for this particular clinical presentation.
\end{abstract}

\section{$(\mathcal{C}$ Clin Pathol 1994;47:858-859)}

In a patient with recurrent thrombotic disease that cannot be explained by disorders in the coagulation system or by vascular, immunological, or metabolic derangements, and with recurrences of thrombosis even during adequately controlled anticoagulant treatment, there is a very high suspicion of the presence of malignant disease. A young woman presented with a dramatic history of recurrent thrombotic occlusions of arteries and veins, occurring over a very short period of time and during high dose anticoagulant treatment. Full physical, laboratory, and imaging investigations failed to reveal any clue for malignant disease during life. Postmortem examination revealed extensively metastatic adenocarcinoma in the vessels of various organs.

\section{Case report}

A 38 year old woman was admitted to the neurology department complaining of acute motor aphasia and right-sided hemiparesis. A computed tomography scan revealed cortical infarction of the brain, due to occlusion of the left middle cerebral artery. She had no history of medical problems and she neither smoked cigarettes nor drank alcohol. She did not use any medication. Physical examination, cardiovascular examination, laboratory tests (including determination of coagulation factors, coagulation inhibitors, immunological and metabolic parameters) did not elucidate a specific cause for the occurrence of the cerebral infarction. The patient recovered well and was discharged with aspirin $80 \mathrm{mg}$ daily. Over the following five months she frequently visited her general practitioner and the outpatient clinic with recurrent thrombophlebitis of superficial veins of both arms and legs. Five months after discharge the patient was readmitted with a recurrent cerebral infarction, due to occlusion of the left posterior cerebral artery. Oral anticoagulant drugs (fenprocoumon) were added to the aspirin regimen and were maintained at an adequate level (International Normalised Ratio $2 \cdot 5-4 \cdot 5$ ). After an initial good recovery and when she was already fully mobilised, she developed thrombosis of proximal and various distal deep veins of the left leg (confirmed by venography). She was treated with therapeutic doses of low molecular weight heparin (Fraxiparin) in combination with the oral anticoagulant drugs and aspirin. Despite this extensive anticoagulant treatment, an acute arterial occlusion of the left superficial femoral artery occurred one month later (confirmed by angiography), followed by the development of a (venographically confirmed) proximal deep venous thrombosis of the right leg and pulmonary embolism, as visualised by high probability ventilation-perfusion scintigraphy. Two months later a thrombotic occlusion of the right superficial femoral and popliteal artery became apparent (visualised by angiography), and to maintain sufficient flow into the threatened right lower limb it was necessary to add the continuous infusion of prostacyclin (Flolan) to the anticoagulant treatment. Another two months later the patient developed a further cerebral infarction of the right hemisphere. After initial improvement, 14 days after this episode she suddenly deteriorated with the occurrence of another left-sided cerebral infarction accompanied by circulatory shock, progressive metabolic derangement, and respiratory insufficiency. She was intubated and ventilated and received circulatory support, but she died a few hours later.

During the early stage of her disease and after exclusion of vascular, coagulative, immunological or metabolic causes of the recurrent thrombosis, the existence of malignant disease was suspected and an extensive series of diagnostic examinations performed. Detailed anamnesis and physical examination did not indicate any abnormality. Radiological, scintigraphic, and endoscopic investigations, including chest $x$ ray picture, mammography, ultrasound examination of upper and lower abdomen and pelvis, computed tomography of neck, chest, abdomen and pelvis, thyroid gland scintigraphy, gallium whole body scintigraphy (oesophageal)echocardiography, laparoscopy with collection and examination of abdominal fluid, endovaginal ultrasound and endoscopy with random biopsy of stomach and colon, failed to reveal evidence of malignant disease. Laboratory screening for markers associated with malignant processes was normal except for a slightly raised CA-125 (38 U/l, and on a 
later occasion $230 \mathrm{U} / \mathrm{l}$; normal value $35 \mathrm{U} / \mathrm{l}$ ). At various times highly sensitive markers for the existence of systemic hypercoagulability were determined. Markers for thrombin generation (prothrombin activation fragment $F_{1+2}$ and thrombin-antithrombin III complexes) and for fibrinogen to fibrin conversion (fibrinogen activation peptide $\mathrm{FpA}$ ) as well as parameters for contact system activation (factor XIIa-C1 inhibitor complexes and kallikrein-C1 inhibitor complexes) were at all times within the normal range, and markers for activity of the fibrinolytic system (plasminogen activator activity, plasmin- $\alpha_{2}$ antiplasmin complexes) were also normal.

\section{Pathological findings}

Postmortem investigation showed manifestations of extensive intravascular thrombosis, with a necrotic first toe of the right foot, due to an occlusive arterial thrombosis in the right femoral artery, an adherent thrombus in the left femoral artery, a non-marantic vegetation on one of the triscupid valve cusps and an old ischaemic lesion in the anteroseptal wall of the left ventricle, without evident coronary pathology.

There was a small atrial septal defect. The lungs showed some congestion and an early pneumonia in the right lower lobe. There was no evidence of previously clinically demonstrated pulmonary embolisms. The carina showed some enlarged lymph nodes. The mucosa of the stomach and duodenum was diffusely haemorrhagic, accompanied by the presence of blood and melaena. Cerebral necropsy showed extensive cerebromalacia in the right frontoparietal region and also in the left frontal area.

Although no gross tumor localisations could be found, microscopic evaluation, however, showed extensive intrapulmonary haematogeneous and lymphogeneous metastasis of a poorly differentiated carcinoma. Intravascular metastases were also found in the liver and the ovary. The lymph nodes in the carina were also extensively invaded by tumour cells. Microscopy of the right medial cerebral artery showed organised thrombus with fibrin and recanalisation. In the lumina of the recanalised vessels clumps of tumour cells were present.

Immunohistochemistry showed tumour cell positivity for the cytokeratin CAM $5 \cdot 2$. The periodic acid Schiff diastase stain demonstrated intracellular mucus production. As no primary localisation of the tumor could be found, it was concluded that an adenocarcinoma of unknown primary site (ACUPS) was present in this patient.

\section{Discussion}

The association between the presence of a malignant disease and the development of thrombosis was established more than one century $\mathrm{ago}^{1}$ and the occurrence of (particularly venous) thrombosis as a first clinical manifestation of cancer has been reported by numerous authors. ${ }^{2-4}$ It was demonstrated that in patients with cancer, systemic activation of blood coagulation, most probably due to procoagulant effects of the tumour, may occur, resulting in a prothrombotic state and thereby explaining the occurrence of thrombosis. ${ }^{56}$

In contrast to the mainly venous thrombotic complications that are normally encountered in cancer patients, in this patient both arterial and venous blood vessels were affected. Furthermore, highly sensitive molecular markers for the detection of hypercoagulability were repeatedly within the normal range and the recurrences of arterial and venous occlusions occurred during multiple anticoagulant treatment. These observations may suggest that in this case the arterial and venous occlusion may have been caused by intravascular localisation of tumour cells with secondary thrombosis rather than primary hypercoagulability resulting in blood clot formation. The capacity of tumours to adhere to vessel walls has been reported before.? However, it is tempting to speculate that this particular clinical presentation might have been due to specific characteristics of the adenocarcinoma cells of this patient, such as the expression of adhesion molecules, specific for endothelial cells, at the surface membrane of the cells. ${ }^{89}$

It is not known whether in patients who present with (recurrent) thrombosis without an obvious cause (such as recent surgery, immobilisation, or trauma) the execution of an extensive programme of laboratory and instrumental examinations for the detection of cancer is useful. ${ }^{2}$ As illustrated in this case, such a diagnostic programme, regardless of how rigorous it is, is frequently not successful and most of the diagnostic investigations carry with them a risk for procedure-related morbidity. Furthermore, it can be debated whether the detection of malignant disease in such patients will eventually increase the potential for (curative) treatment of the cancer and ultimately result in lower mortality. In our patient the adenocarcinoma was extensively metastatic throughout various parts of the body. However, its intravascular localisation may explain why it was not detected when the patient was alive, despite extensive searching. It can only be speculated that highly sensitive cytological examination of the circulating blood using specific markers for malignant cells might have detected the malignant cells in this particular case.

Trousseau A. Phlegmasia alba dolens. Clinique Médicale de l'Hôtel-Dieu de Paris. London: The New Sydenham l'Hotel-Dieu de Paris.

2 Prandoni P, Lensing AWA, Büller HR, et al. Deep vein thrombosis and the incidence of subsequent symptomatic cancer. $N$ Engl f Med 1992;327:1128-33.

3 Goldberg RJ, Seneff M, Gore JM, et al. Occult malignan neoplasm in patients with deep vein thrombosis. Arch In Med 1987;147:251-3.

4 Silverstein RL, Nachman RL. Cancer and clottingTrousseau's warning. N Engl f Med 1992;327:1163-4.

5 Dvorak HF. Abnormalities of hemostasis in malignancy. In: Colman RW, Hirsh J, Marder VJ, et al. Hemostasis and thrombosis: basic principles and clinical practice. Philadelphia: JB Lippincott Company, 1982:1143-57.

6 Pineo GF, Regoeczi E, Hatton MWC, Brain MC. The activation of coagulation by extracts of mucus: a possible activation of coagulation by extracts of mucus: a possible pathway of intravascular coagulation accompanyin adenocarcinomas. F Lab Clin Med 1973;82:255-66.

Warren BA, Vales $\mathrm{O}$. The adhesion of thromboplastic tumour emboli to vessel walls in vivo. Br $\mathcal{F}$ Exp Patho

8 Honn KV, Tang DG. Adhesion molecules and tumor cell interaction with endothelium and subendothelial matrix. interaction with endothelium and subendo

9 Johnson JP. Cell adhesion molecules in neoplastic disease. Int $\mathcal{F}$ Clin Lab Res 1992;22:69-72. 